\title{
Pulse Extinction Ratio Improvement Using SPM in an SOA for OTDM Systems Applications
}

Nielsen, Mads Lønstrup; Olsson, B.-E.; Blumenthal, D. J.

Published in:

I E E E Photonics Technology Letters

Link to article, DOI:

$10.1109 / 68.980536$

Publication date:

2002

Document Version

Publisher's PDF, also known as Version of record

Link back to DTU Orbit

Citation (APA):

Nielsen, M. L., Olsson, B-E., \& Blumenthal, D. J. (2002). Pulse Extinction Ratio Improvement Using SPM in an SOA for OTDM Systems Applications. I E E E Photonics Technology Letters, 14(2), 245-247.

https://doi.org/10.1109/68.980536

\section{General rights}

Copyright and moral rights for the publications made accessible in the public portal are retained by the authors and/or other copyright owners and it is a condition of accessing publications that users recognise and abide by the legal requirements associated with these rights.

- Users may download and print one copy of any publication from the public portal for the purpose of private study or research.

- You may not further distribute the material or use it for any profit-making activity or commercial gain

- You may freely distribute the URL identifying the publication in the public portal 


\title{
Pulse Extinction Ratio Improvement Using SPM in an SOA for OTDM Systems Applications
}

\author{
Mads L. Nielsen, Student Member, IEEE, Bengt-Erik Olsson, and Daniel J. Blumenthal, Senior Member, IEEE
}

\begin{abstract}
Improvement of pulse extinction ratio from 15-20 dB to approximately $40 \mathrm{~dB}$ of a 10-GHz pulse train is demonstrated using the self-phase modulation-induced spectral shift caused by a semiconductor optical amplifier (SOA). The improvement permits penalty-free optical time-division multiplexing to $40 \mathrm{~Gb} / \mathrm{s}$, and demultiplexing back to $10 \mathrm{~Gb} / \mathrm{s}$ of an intentionally distorted signal. Without using the SOA the $40-\mathrm{Gb} / \mathrm{s}$ signal is not receivable.
\end{abstract}

Index Terms-Extinction ratio, self-phase modulation, semiconductor optical amplifier.

\section{INTRODUCTION}

$\mathbf{O}$ PTICAL time-division multiplexing (OTDM) is an efficient way of increasing the bitrate in transmission systems and optical networks. The OTDM transmitters, as well as channel add/drop units, require time interleaving of short data pulses, which in turn requires that the interleaving channels contain very little energy outside their designated bit-slots. Thus, the pulse extinction ratio (ER) has to be very large to avoid interference between adjacent channels. The pulse ER is here defined as the ratio between the peak pulse power and the maximum power of the unwanted background signal between the pulses. In a practical transmitter, one pulse source is usually split into a number of channels and subsequently modulated with data. All channels are then time interleaved to accomplish a high bit-rate OTDM train. Imperfections of the pulse source, as e.g., mode hopping between neighboring cavity modes or a limited coherence time, can result in amplitude fluctuations in the OTDM signal if the pulse ER is not sufficiently high. This has been identified as an important source of performance degradation in OTDM systems [1]. In most published OTDM experiments, a mode-locked fiber ring laser is used as a pulse source, which usually has a very good pulse ER $(>40 \mathrm{~dB})$. However, in practice, it would be better if a cheap and compact pulse source could be used, e.g., a $\mathrm{LiNbO}_{3}$ modulator or an electroabsorption modulator (EAM). Unfortunately, these pulse sources usually have a poor pulse ER, typically below $15-25 \mathrm{~dB}$, which limits their use in OTDM systems. Several schemes to improve the pulse ER through intensity discrimination have been

Manuscript received April 6, 2001; revised October 9, 2001

M. L. Nielsen is with Research Center COM, Technical University of Denmark, DK-2800 Lyngby, Denmark.

B.-E. Olsson was with the Optical Communications and Photonics Networks Laboratory, Department of Electrical and Computer Engineering, University of California Santa Barbara, Santa Barbara, CA 93106 USA. He is now with Optillion AB, 41136 Göteborg, Sweden.

D. J. Blumenthal is with the Optical Communications and Photonics Networks Laboratory, Department of Electrical and Computer Engineering, University of California Santa Barbara, Santa Barbara, CA 93106 USA.

Publisher Item Identifier S 1041-1135(02)00247-1.

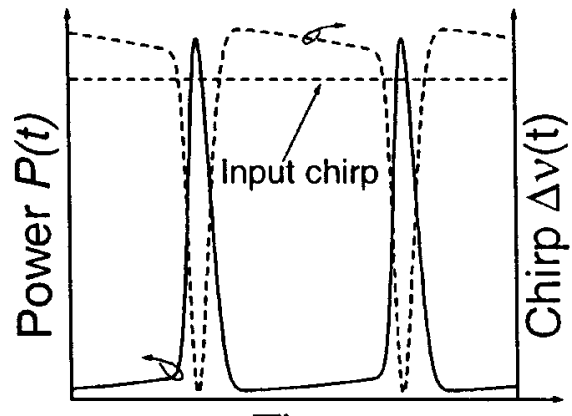

Time $t$

Fig. 1. Output pulse shape (solid), and corresponding chirp (dashed)

suggested, but most rely on SPM in a nonlinear fiber [2], [3], which is very power consuming, inherently bulky, and not integrable. In this letter, we investigate the effects of a poor pulse ER on a 40-Gb/s OTDM system, and present a new way of increasing the pulse ER, and consequently the system performance using self-phase modulation (SPM) in a semiconductor optical amplifier (SOA), which is compact and integrable. We report penalty-free $40-\mathrm{Gb} / \mathrm{s}$ data reception after pulse ER improvement of the original $10-\mathrm{GHz}$ signal from $15-20 \mathrm{~dB}$ to approximately $40 \mathrm{~dB}$, which is required for time interleaving four OTDM channels with negligible penalty due to interference [4]. The experiment is supported by numerical simulations. The results indicate a potential for using this scheme with e.g., an EAM integrated with an SOA.

\section{BASIC PRINCIPLE}

Pulses with sufficiently high energy propagating through an SOA are chirped as a result of saturation-induced SPM [5]. It can be shown that the temporal variation of the frequency chirp approximately follows the pulse shape [5]. This is illustrated in Fig. 1, where the solid curve shows the power of two pulses with finite ER at the output of an SOA, and the dashed curve shows the corresponding chirp. The horizontal dashed line indicates the (nonexistent) chirp of the pulses at the input of the SOA. From Fig. 1, it is obvious that the SOA translates pulse power into spectral shift, which inevitably leads to spectral broadening. Fluctuations in the peak power of the input pulses will not, however, result in fluctuations in the spectral shift, since the SOA is operated in deep saturation. It is clear from Fig. 1, that the broadening occurs mainly on the low-frequency (the red) side, because the increase in phase caused by the stimulated recombination is much faster than the decrease due to gain recovery. These features are seen more clearly in 


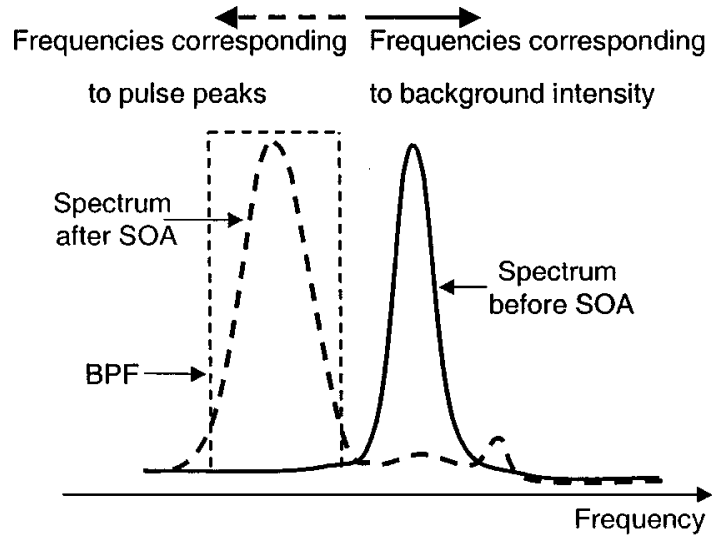

Fig. 2. Schematic of optical pulse spectrum at input (solid) and output (dashed) of SOA.

TABLE I

SIMULATION DATA

\begin{tabular}{l|l||l|l}
\hline$J\left(\mathrm{kA} / \mathrm{cm}^{2}\right)$ & 30 & $d(\mu \mathrm{m})$ & 0.33 \\
\hline$A\left(\mathrm{~s}^{-1}\right)$ & $1.0 \cdot 10^{8}$ & $L(\mathrm{~mm})$ & 1.0 \\
\hline$B\left(\mathrm{~m}^{3} \mathrm{~s}^{-1}\right)$ & $9.0 \cdot 10^{-16}$ & $\alpha_{\text {int }}\left(\mathrm{m}^{-1}\right)$ & $2.0 \cdot 10^{3}$ \\
\hline$C\left(\mathrm{~m}^{6} \mathrm{~s}^{-1}\right)$ & $5.0 \cdot 10^{-41}$ & $\Gamma$ & 0.40 \\
\hline$a\left(\mathrm{~m}^{2}\right)$ & $3.0 \cdot 10^{-20}$ & $\beta$ & 7.0 \\
\hline
\end{tabular}

Fig. 2, where the input (solid) and output (dashed) spectra are illustrated. The power-dependent spectral shift results in a separation of the high-intensity content of the pulses, corresponding to the peaks from the unwanted background power. Consequently, filtering out the red-shifted part of the spectrum with a bandpass filter (BPF), as indicated by the dashed box, the pulse ER can potentially be increased. Depending on the filtering, the spectral shift can also be accompanied by pulse compression, since the red-shifted peak is usually broader than the input spectrum [5].

\section{SimULATIONS}

To verify the principle outlined above, pulse propagation through an SOA has been modeled. The device is divided into $M$ sections to account for longitudinal effects. The time evolution of the carrier density $N$, the optical power $P$, and the phase $\phi$ of the pulse field-envelope $A(z, t)=\sqrt{P(z, t)} \exp (j \phi(z, t))$ is described by solving the carrier rate equation, and the field propagation equation in all the sections [5]. Parameters used in the simulations are shown in Table I, where $J$ is the injection current density, $L$ and $d$ are the length and height of the active region, respectively, $R(N)=A N+B N^{2}+C N^{3}$ is the spontaneous recombination rate, $a$ is the differential gain (we assume the gain is linear in $N), \Gamma$ is the optical confinement factor, $\alpha_{i n t}$ is the internal scattering loss coefficient, and $\beta$ is the linewidth enhancement factor. Introducing the Fourier- and inverse Fourier transform operators $\widetilde{F}$ and $\widetilde{F}^{-1}$, respectively, the signal power $P_{H}(t)$ after filtering can be expressed as

$$
P_{H}(t)=\left|\widetilde{F}^{-1}\{\widetilde{F}[A(L, t)] H(f)\}\right|^{2}
$$

where $H(f)$ is the BPF response.

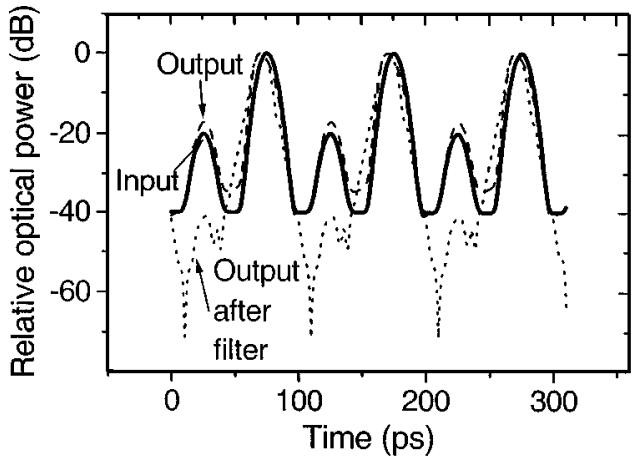

Fig. 3. Simulated pulse train at input (solid) of SOA, after SOA (dashed), and after SOA + BPF (dotted).

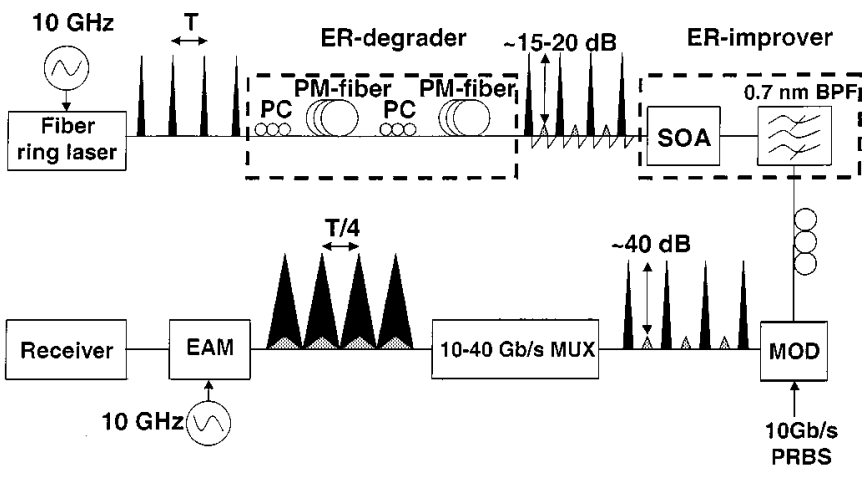

Fig. 4. Schematic of experimental setup.

The input signal to the SOA is a $10-\mathrm{GHz}$ pulse train of 12-ps wide (FWHM) Gaussian pulses with a pulse ER of $40 \mathrm{~dB}$ and an average power of $5 \mathrm{dBm}$. As shown by the solid curve in Fig. 3, a weak pulse suppressed by $20 \mathrm{~dB}$ is placed between every two signal pulses. This reduces the effective pulse ER to $20 \mathrm{~dB}$. This rather unusual way of degrading the ER is chosen because a similar approach is used in the experiment presented below. The dashed line in Fig. 3 shows the pulse train at the output of the SOA, and the ER is observed to have decreased slightly due to gain saturation. This signal is passed through a Lorentzian BPF with a FWHM bandwidth of $0.5 \mathrm{~nm}$. The peak of the filter is offset $0.8 \mathrm{~nm}$ to the red (low frequency) side of the spectrum, measured from the peak of the input spectrum (see Fig. 2). The result is shown by the dotted curve in Fig. 3, and an improvement of the ER to approximately $40 \mathrm{~dB}$ is observed.

\section{EXPERIMENT}

The 40-Gb/s OTDM system is shown in Fig. 4. The pulse source is an Er-doped fiber ring laser, generating a 10-GHz pulse train with an ER of approximately $40 \mathrm{~dB}$. The pulses enter an "ER-degrader," which consists of a polarization controller (PC), a piece of polarization maintaining (PM) fiber followed by another PC, and yet another piece of PM fiber. The differential group velocity delays (DGD) between the two principal axes of the PM-fiber are 150 and $75 \mathrm{ps,} \mathrm{respectively,} \mathrm{corresponding}$ to 1.5 and $0.75 T$, where $T$ is the $10-\mathrm{Gb} / \mathrm{s}$ timeslot of $100 \mathrm{ps}$. The PC at the input of the "ER-degrader" aligns the polarization with one principal axis of the first PM fiber, and the second PC 
does the same for the second piece of PM fiber. At the output of the "ER-degrader," we have the same signal as at the input, except for three very weak pulses with alternating polarization following each original pulse. These pulses arise because of imperfect alignment of the polarization. Since the pulses in the polarization state orthogonal to the signal will be suppressed by a polarizer built into the data-modulator, only the weak pulse centered between the signal pulses will participate in the ER-degradation. We estimate the pulse ER at the output of the "ER-degrader" to be around $15-20 \mathrm{~dB}$. Inside the $10-40-\mathrm{Gb} / \mathrm{s}$ multiplexer, which is a passive fiber-based pulse interleaver based on three 3-dB power splitters used to simulate an OTD-multiplexer, the signal pulses and the weak ER-degrading pulses end up on top of each other. This will cause the pulses to interfere either constructively or destructively, depending on the phase relationship between them. In the present case, the signal from the ring laser makes phase-hops on a time scale of about 1-10 s, which shows up in the multiplexed $40-\mathrm{Gb} / \mathrm{s}$ data stream as large amplitude fluctuations. We attribute this behavior to mode hopping between neighboring cavitymodes, which results in a change of the optical path length, and consequently the phase difference between interfering signals.

The pulsewidth after the "ER-improver," consisting of the SOA and the BPF, was approximately $9 \mathrm{ps}$, compared to $12 \mathrm{ps}$ at the input. After the filtering, the pulses are modulated with a pseudorandom bit sequence (PRBS) of pattern length $2^{31}-1$. The modulated pulse train enters the multiplexer, and because the pulse ER has been significantly improved in the SOA, the amplitude fluctuations are greatly reduced. The $40-\mathrm{Gb} / \mathrm{s}$ signal is demultiplexed using an EAM, and the demultiplexed $10-\mathrm{Gb} / \mathrm{s}$ signals are received and evaluated through bit error-rate (BER) measurements.

In the inset of Fig. 5, the eye diagram of the 40-Gb/s signal with (lower) and without (upper) the aid of the "ER improver" is showed, and the improvement is clear. Because the interference varied substantially over time, it was impossible to measure stable BERs without using the "ER-improver." As illustrated in Fig. 5 where the BER performance of the entire system is compared to the back-to-back performance, the "ER-improver" completely restores the pulse quality and removes the interference problems. These problems are generally caused both by pulse overlap and by a finite pulse ER [1], but in this experiment the latter is the most dominant. This was verified by the fact that the system performance without the "ER-improver" did not improve by decreasing the input pulsewidth from 12 to $8 \mathrm{ps}$. This proves that the dramatic improvement obtained by using the SOA is not due to pulse compression.

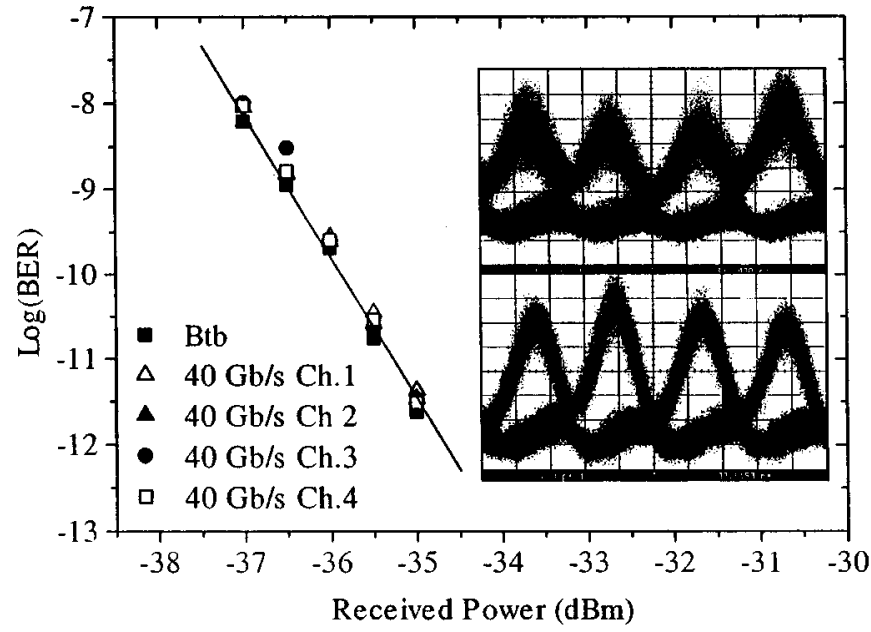

Fig. 5. BER performance of the four demultiplexed channels, compared to $10 \mathrm{~Gb} / \mathrm{s}$ back-to-back. Insets: 40-Gb/s eye diagram without (upper), and with (lower) the aid of the "ER-improver."

\section{CONCLUSION}

We proposed a scheme to increase the pulse ER using SPM in an SOA and verified the principle numerically and experimentally. By purposely degrading the ER of a $10-\mathrm{Gb} / \mathrm{s}$ signal from a fiber ring laser from approximately $40 \mathrm{~dB}$ to $15-20 \mathrm{~dB}$ before multiplexing to $40 \mathrm{~Gb} / \mathrm{s}$, we found it impossible to detect the data. Using the proposed principle, we were able to multiplex to $40 \mathrm{~Gb} / \mathrm{s}$ and demultiplex back to $10 \mathrm{~Gb} / \mathrm{s}$ with no penalty. We have verified that the pulse ER is very critical in OTDM systems, and shown an example of simple signal processing in an SOA. We believe that the approach will be equally successful if applied to pulses from a modulator with relatively low ER, such as a $\mathrm{LiNbO}_{3}$ modulator or an EAM provided that they can give sufficiently short pulses $(<12 \mathrm{ps})$ to avoid serious pulse tail overlap.

\section{REFERENCES}

[1] A. D. Ellis, T. Widdowson, I. D. Phillips, and W. A. Pender, "High speed OTDM networks employing electro-optic modulators," IEICE Trans. Electron., vol. E81-C, pp. 1301-1308, Aug. 1998.

[2] H. P. Sardesei and A. M. Weiner, "Nonlinear fiber-optic receiver for ultrashort pulse code division multiple access communications," Electron. Lett., vol. 33, pp. 610-611, Mar. 1997.

[3] J. Yu, A. Clausen, H. N. Poulsen, P. Jeppesen, X. Zheng, and C. Peucheret, " $40 \mathrm{~Gb} / \mathrm{s}$ wavelength conversion in a cascade of an SOA and a NOLM and demonstration of extinction ratio improvement," Electron. Lett., vol. 36, pp. 963-964, May 2000.

[4] K. S. Jepsen, H. N. Poulsen, A. T. Clausen, and K. E. Stubkjr, "Investigation of cascadability of add-drop multiplexers in OTDM systems," in Proc. ECOC'98, vol. 1, 1998.

[5] G. P. Agrawal and N. A. Olsson, "Self-phase modulation and spectral broadening of optical pulses in semiconductor laser amplifiers," IEEE J. Quantum Electron., vol. 25, pp. 2297-2306, Nov. 1989. 